\title{
Maeda-Dadhich Solutions as Real Black Holes
}

\author{
S. O. Alexeyev ${ }^{1, \star}, A$. N. Petrov ${ }^{1, \star \star}$, and B. N. Latosh ${ }^{2,3, \star \star \star}$ \\ ${ }^{1}$ Sternberg Astronomical Institute, Lomonosov Moscow State University, Universitetsky Prospekt, 13, \\ Moscow 119991, Russia \\ ${ }^{2}$ Faculty of Natural and Engineering Science, Dubna International University, Universitetskaya Str., 19, \\ Dubna, Moscow Region, Russia \\ ${ }^{3}$ Bogoliubov Laboratory of Theoretical Physics, Joint Institute for Nuclear Research, Joliot-Curie 6, 141980 \\ Dubna, Moscow region, Russia
}

\begin{abstract}
A four-dimensional static Schwarzschild-like solutions obtained in [4, 5] in the frames of the Einstein-Gauss-Bonnet gravity at the Kaluza-Klein split are analyzed. In such models matter is created by auxiliary dimensions. The main goal of our work is to check that these solutions are physically sensible, and to examine their characteristics, which could be observable.
\end{abstract}

\section{Introduction}

String/M-theory [2] is still a perspective candidate for the unified theory of all physical interactions, but a problem of its experimental verification remains unsolved. However, the generic formulation of a string theory is not completed and in some cases it is appropriate to consider the low energy approximation. The second order expansion is the Gauss-Bonnet one. In the framework of EinsteinGauss-Bonnet (EGB) gravity a set of interesting solutions has been obtained, especially black hole ones [3]. One can use the results of their physical effects to test the string theory and to search for the new physics in gravitation. In the present study we are looking for such effects in the solution obtained in the Maeda and Dadhich (MD) papers [4] devoted to EGB gravity with a cosmological constant. Of course, basic properties of the MD solutions were analyzed carefully by Maeda and Dadhich [4]. On the other hand, properties which could be useful for experimental/observational physics were not considered completely. Thus, it is possible to study physically sensible properties and characteristics, which could be observable.

The main assumption for the MD solutions is the Kaluza-Klein type splitting of the EGB spacetime. So, there is a physical (dynamic) space-time (of 2, 3 or 4 dimensions) and a space of auxiliary dimensions. The later one has a constant curvature with the negative sign, whereas in the standard Kaluza-Klein model a space-time of auxiliary dimensions is flat. We concentrate on the 4-dimensional static Schwarzschild-like solution because it seems to be more promising in searching for physically sensible effects. For simplicity we restrict ourselves to the minimal case of 6D EGB space-time that

\footnotetext{
^e-mail: alexeyev@sai.msu.ru

$\star \star$ e-mail: alex.petrov55@gmail.com

$\star \star \star$ e-mail: latosh.boris@gmail.com
} 
has been studied separately by Molina and Dadhich [5] and we denote as DM. The EGB solution properties in 6D are similar to those in arbitrary EGB dimensions with $N>6$.

We check the DM solutions from two points of view. To be physically sensible these solutions have to have, first, acceptable theoretical description, and, second, observable evidences, at least, principally. Theoretical description has to give a non-contradictive definitions of important characteristics. Undoubtedly, for solutions, which pretend to present astrophysical gravitating objects, such a characteristic is a total mass of the system. Not less important theoretical property is a stability of the solution. We notice that a stability is directly connected with a well defined total mass, and independently we state a possibility that the DM solutions to leave stable. Thus, chances to detect related astrophysical objects arise.

Properties, which could be potentially observable and which we study are orbital and thermodynamic effects related to the DM solutions. The last part of the paper is just devoted to these problems. As known, thermodynamic properties are directly connected with a horizon structure of solutions. As it turns out, geometrical structure of the DM solutions is quite non-trivial and we examine it in a significantly more detail than it is given in [4]-[5].

\section{Dadhich-Molina solution}

The restriction of the EGB gravity in 6D in the DM solution [5] leads to the following form of action:

$$
S=\frac{1}{2 \kappa_{6}} \int d^{6} x \sqrt{-g}\left(R-2 \Lambda+\alpha L_{G B}\right)+S_{\text {matter }} .
$$

Here and below we use $c=\hbar=1$ units, $\kappa_{6}$ is the 6-dimensional gravitational (Einstein) constant, $\alpha>0$ is the Gauss-Bonnet coupling constant; $R$ is a 6-dimensional Ricci scalar, $\Lambda$ is the cosmological constant and $L_{G B}$ is the Gauss-Bonnet term:

$$
L_{G B}=R^{2}-4 R_{\mu \nu} R^{\mu \nu}+R_{\mu \nu \rho \sigma} R^{\mu \nu \rho \sigma} .
$$

The main MD assumptions are as follows:

- The space-time of EGB gravity (6D in our case) is homeomorphical to $M^{4} \times K^{2}$ where $M^{4}$ is a 4-dimensional physical space-time and $K^{2}$ is a space of constant curvature with radius $r_{0}$ and sign $\bar{k}$ (they are not determined now). It is just the Kaluza-Klein type splitting.

- EGB vacuum case $S_{\text {matter }}=0$ is considered.

- Model parameters is constrained by the following relation:

$$
\bar{k} / r_{0}^{2}=-1 / 4 \alpha=\Lambda,
$$

Under such assumption the original solution was obtained [4].

Black hole solution obtained in [4] have the following form

$$
\begin{gathered}
d s^{2}=f_{ \pm} d t^{2}-\frac{d r^{2}}{f_{ \pm}}-r^{2}\left(d \theta^{2}-\sin ^{2} \theta d \varphi\right), \\
f_{ \pm}=1+\frac{r^{2}}{4 \alpha}\left[1 \pm \sqrt{\frac{2}{3}+16\left(\frac{\alpha^{\frac{3}{2}} M}{r^{3}}-\frac{\alpha^{2} q}{r^{4}}\right)}\right],
\end{gathered}
$$

where the integration constants $M$ and $q$ appear to be the main solution parameters. Historically, $M$ is treated as a mass of a black hole and $q$ is an additional charge. 


\section{Total Mass}

Energy connected characteristics play crucial role in a description of potentially observable physical objects. So, first of all, we have to define and calculate total mass (energy) of the solution (4)-(5). The mass of the solution may be calculated via superpotential technique. Various forms of superpotentials in $D>4$ dimensions, applicable in AdS and AdS-like backgrounds, have been constructed both in general relativity (GR) and in EGB theory, see, for example, [6]-[10] and references there in.

Propper calculation were made in paper [1] and the total mass of the solution given by the formula

$$
\mathcal{E}= \pm \sqrt{\frac{3}{2}} \alpha^{1 / 2} M
$$

A system is physically sensible if its total energy is not negative $\mathcal{E} \geq 0$, therefore one must set $\pm M_{\mathrm{eff}} \geq 0$. Furthermore, the charge parameter $q$ does not contribute into $\mathcal{E}$, so we have an analogy with the Reisner-Nordsröm-AdS black hole, where $\mathcal{E}=M$, and $Q^{2}$ does not contribute into $\mathcal{E}$.

\section{Stability}

The problem of DM solution stability is important from a theoretical point of view and is crucial in cosmological and astrophysics applications. Usually, for such test one perturbs metric coefficients and checks their further behavior. Technically this is carried out by perturbing initial field equations and solving them. However, to obtain the DM solution special restrictions and conditions have been used. The conditions state that curvature of auxiliary dimensions is constant, perturbations propagating in auxiliary dimensions may violate the condition. Without the condition Maeda-Dadhich metric no longer solve field equations and thus may not be treated as proper background for perturbations. Thus we consider only four-dimensional perturbations of Maeda-Dadhich solution.

It is constructive to consider two cases: when (i) axial symmetry is applied, (ii) there are no symmetries. The case (i) means the existence of perturbations with axial symmetry, corresponding to black holes with small angular momentum. This case is quite important because the MD model excludes rotating black holes as it is stated in [4]-[5]. Further, the instability of the DM metric with axial perturbations means that the solution must be destroyed by a rotation. Such a conclusion could be fatal for cosmological and astrophysics applications where most of objects rotate.

The detail discussion of the task may be found in paper [1]. In the paper it was shown that in both cases (i) and (ii) perturbations admit solution exponentially dumping in time, and, thus, the DM solution has a possibility to conserve a stability.

\section{Geometrical Structure}

The geometrical structure of the solution is important because solution may describe unphysical objects plagued with singularities. Thus the geometrical structure is crucial research topic. As the DM solution is stable, we analyze its geometrical structure up to $r \rightarrow 0$. For the sake of simplicity hereafter we work within $c=\hbar=\alpha=1$ units. Then $f_{ \pm}$defined in (5) is rewritten as

$$
f_{ \pm}(r)=1+\frac{r^{2}}{4}\left[1 \pm \sqrt{\frac{2}{3}+16\left(\frac{M}{r^{3}}-\frac{q}{r^{4}}\right)}\right] .
$$

We call $f_{+}$and $f_{-}$positive and negative branches of the solution, respectively. We also will consider only objects with positive energy. 
In paper [1] more detail analysis is given. In the paper we were able to obtain the diagram describing all possible configurations described by Maeda-Dadhich solution. The diagram is given on Fig. 2. The left hand side of the diagram describe positive branch of the solution, and right hand side - negative branch of the solution. In forbidden regions solution have region with complex metric and the region is not covered by event horizon, thus such regions provide nonphysical solutions. Positive branch of the solution can describe only regular naked singularities and may not provide a description of a black hole. Negative branch, on the other hand, may provide description of bath naked singularity and black hole. It is crucial to highlight that negative branch of the solution have a region with a complex metric for any $q>0$, but the region is cover by an event horizon.

Thus Maeda-Dadhich solution may provide regular description of a naked singularity and of a black hole, but also describes black holes plagued with singularities.

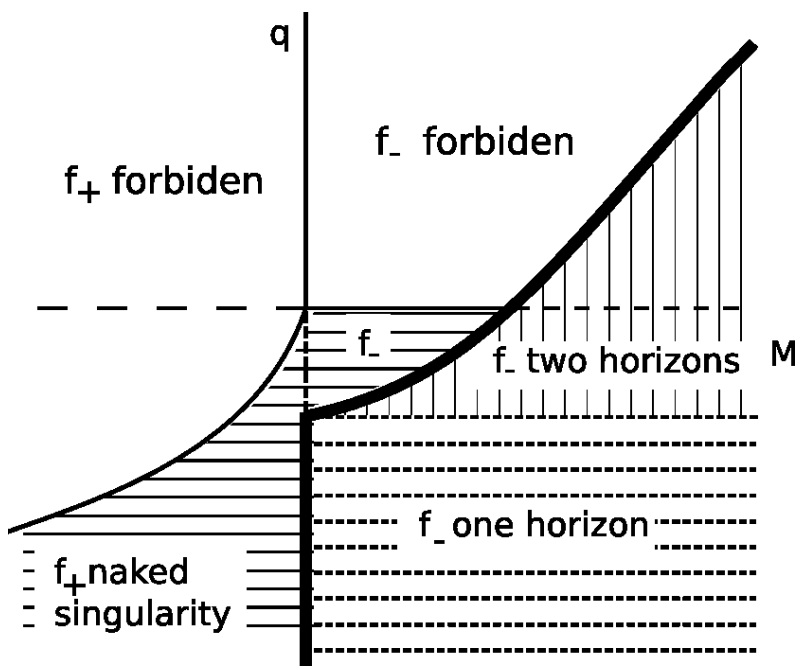

Figure 1. Dadhich-Molina solution phase diagram

\section{Orbital effects}

Orbital effects play an important role in black hole-like solutions, as they are coupled with an accretion picture that is actually observed [14]. We assume cosmological and astronomical scales and, therefore, can use several approximations. We assume that solution has a mass of stellar range or higher, hence one may work with series expansion of a metric. But that case is reducing to well-know DMPR solution [12]. The solution was studed in series of papers [13, 15-17]

Because the MD metric is static and spherical symmetric the standard method presented in the Chandrasekhar book [11] can be applied effectively to study stable orbits. Already, this method has been used in [13] for examining the Dadhich-Rezania solution [12] that has many similar properties with the DM solution. Therefore, not repeating here the derivation of an application of the Chandrasekhar method, we rather compare the results of its application to both the DM solution and the Dadhich-Rezania solution and the critical dependence between $q$ and $M$ was established

$$
|q| \leq \frac{9}{8 \sqrt{6}} M^{2}
$$


If $q$ is bigger than $\frac{9}{8 \sqrt{6}} M^{2}$, test particles orbits change [17] as the quasinormal modes for the scattering [16] and the shadow size of the black hole [15]. Engaging the recent data on M87 galaxy [18], we see that there is no evidence in Dadhich-Rezania model manifestation, so the real difference between the DM solution and the Schwarchild one is negligible small for existing accuracy level.

\section{Thermodynamics and Evaporation}

Hawking radiation is the other important effect that provides an instrument for model testing at cosmological scales. We use both the Hawking method [19] and the Shankaranarayanan-PadmanabhanSrinivasan (SPS) one $[20,21]$. An interesting particularity of the discussed model is that the temperature grows with $M$ parameter increasing. Similar behavior takes place in Schwarzschild-AdSlike solution [22], but for sufficiently big masses only. If the mass is small, the temperature of the Schwarzschild-AdS-like black hole decreases with increasing mass. Note that there is the temperature area that is unreachable for black holes. However, the DM solution temperature only increases with increasing mass, the temperature can achieve arbitrary values. More detail discussion of the problem may be found in [1].

In [1] it was shown that thermodynamically stable configurations either would be plagued with singularities or would have zero energy. Also it was shown that the evaporation strongly depends upon the initial charge $q$. Until one estimates the charge of the DM black hole, it is impossible to find the black holes endpoint mass after the evaporation.

\section{Conclusions}

In this work we studied Maeda-Dadhich solution obtained in the framework of $N>4$ Einstein-GaussBonnet gravity. We proved the solution stability under linear perturbations, therefore, Maeda-Dadhich metrics is valid for the description of real astrophysical black holes. We investigated the existence of the solution in positive mass region that was not mentioned in the original papers $[4,5]$. We found additional limitations on solution parameters. The positive branch is valid only for a naked singularity description, while the negative one could describe a black hole with one or two horizons some of which is plagued with singularities. We established that the black hole temperature is not governed by Hawking evaporation law and this fact causes additional limitations on the Maeda-Dadhich black hole. Unfortunately, the thermodynamically stable configurations either have zero energy or plagued with singularities. Thus we concluding that Maeda-Dadhich solution is not the best candidate for astrophysical black hole.

\section{Acknowledgments}

This work was partially supported by individual grants from Dmitry Zimin Foundation "Dynasty" (S.A. \& B.L.). We are grateful to Naresh Dadhich for explanation of results in [4, 5]. We also would like to thank Alexander Zakharov and Alexander Shatskiy for useful discussions. At last, we thank Uliana Voznaya and Deepak Baskaran for the help with improving English.

\section{References}

[1] S. O. Alexeyev, A. N. Petrov and B. N. Latosh, Phys. Rev. D 92, no. 10, 104046 (2015) doi:10.1103/PhysRevD.92.104046 [arXiv:1503.06780 [gr-qc]]. 
[2] John H. Schwarz, String Theory and M-Theory. In "100 Years of Subatomic Physics", Word Scientific, pp. 519-550 (2013).

[3] S.Alexeyev and M.Ponazanov, Phys.Rev. D 55, 2110 (1997); S. O. Alexeyev, K. A. Rannu, JETP 114, 406 (2012)

[4] H. Maeda and N. Dadhich, Phys. Rev. D 74, 021501(R) (2006); hep-th/0605031.

[5] A. Molina and N. Dadhich, Int. J. Mod. Phys. D 18, 599 (2009); arXiv:0804.1194 [gr-qc].

[6] L.F. Abbott and S. Deser, Nucl. Phys. B 195, 76 (1982).

[7] S. Deser and B. Tekin, Phys. Rev. D 67084009 (2003); hep-th/0212292.

[8] N. Deruelle, J. Katz and S. Ogushi, Class. Quantum Grav. 21, 1971 (2004); gr-qc/0310098.

[9] A.N. Petrov, Class. Quantum Grav. 26, 135010 (2009); arXiv:0905.3622 [gr-qc].

[10] A.N. Petrov and R.R. Lompay, Gen. Relat. Grav. |bf 45, 545 (2013); arXiv:1211.3268 [gr-qc].

[11] S. Chandrasekhar, The Mathematical Theory of Black Holes, Clarendon Press, 1998.

[12] N. Dadhich, R. Maartens, P. Papadopoulos and V. Rezania, Phys. Lett. B 487, 1 (2000); hepth/0003061.

[13] S.O. Alexeev, D.A. Starodubceva, JET 138, 652 (2010).

[14] Shakura, N.I. and Sunyaev, R.A., Astronomy and Astrophysics, 24, 337-355, 1973

[15] A. F. Zakharov, Phys. Rev. D 90, no. 6, 062007 (2014) [arXiv:1407.7457 [gr-qc]].

[16] C. Chirenti, A. Saa, J. Skakala, Phys. Rev. D 86, 124008 (2012).

[17] D. Pugliese, H. Quevedo, R. Ruffini, Phys. Rev. D 83, 024021 (2011).

[18] S. S. Doeleman et al., Science 338, 355 (2012).

[19] S.W. Hawking, Commun. Math. Phys. 43, 199 (1975)

[20] K. Srinivasan and T. Padmanabhan, Phys. Rev. D 60, 024007 (1999); gr-qc/9812028.

[21] S. Shankaranarayanan, T. Padmanabhan and K. Srinivasan, Class. Quant. Grav. 19, 2671 (2002); gr-qc/0010042.

[22] J. Crisostomo, R. Troncoso and J. Zanelli, Phys. Rev. D 62, 084013 (2000); hep-th/0003271. 